\title{
CASINO a Powerful Simulation Tool for Cathodoluminescence Applications
}

\section{Drouin}

${ }^{1}$ CRN2 (Centre de recherche en nanofabrication et nanocaractrisation), Departmenet de génie électrique et génie informatique, Université de Sherbrooke, Sherbrooke, Québec, J1K 2R1, Canada.

The topic of this paper is to present a powerful tool for SEM analysis. The Monte Carlo simulation program CASINO (www.gel.usherb.ca/casino) has been adapted for CL applications with added absorbed energy distributions and flexibility to models more realistic SEM samples. Simple geometry such as vertical and horizontal planes used in the previous version limits considerably the range of applications of this tool. This 3-D version of the tool is able to simulate electron trajectories of more complex sample geometry such as one shown in Figure 1. The program is using complex algorithms to track down the electron position as it travels within the sample. A tree like algorithm is first used to subdivide the samples, composed of a large number of triangles, in cubes containing a few of them. This technique reduces considerably the computing time needed to locate the position of an electron collision in the sample. Each triangle composing the sample defines a boundary between different regions of different chemical composition. As the electron travels trough those regions the electron mean free path is corrected. The core of the electron trajectory computation is based on a single scattering model. Tabulated Mott elastic cross-sections are used due to fast calculation and accurate interaction at low beam energy. A continuous energy loss model is implemented based on the modified version of Bethe law by Joy and Luo.

The sample presents in Figure 1 is composed of InAs quantum dots (QD) embedded in a InP substrate. Figure 2 shows a cross section schematic of two QDs. Each QD have a diameter of $10 \mathrm{~nm}$ and are buried $70 \mathrm{~nm}$ below the surface sample. Also to simulate the effect of carrier diffusion in the substrate, larger concentric spheres are positions around each QD. The diameter of those diffusion spheres are set to $20 \mathrm{~nm}$ with increasing steps of $10 \mathrm{~nm}$. In order to avoid primary electron interaction with the diffusion rings, the chemical composition is set as the same as the InP substrate. Once the sample configured within CASINO, the users can set the SEM parameters according to experimental conditions used. Figure 3, shows a series of absorbed energy images simulated with primary electrons energy ranging from 2 to $10 \mathrm{keV}$. The intensity image is created by plotting the amount of absorbed energy in InAs QDs with a scale ranging from black meaning no absorbed energy to white been the maximum value. For each image, the total field of view is $1 \mu \mathrm{m}$, the beam diameter is fixed to $10 \mathrm{~nm}$, an array of 50x50 simulations points was used and the number of electron trajectories per points was fixed to 2000. From this set of images, a clear decreased in image resolution can be observed as the energy is increased from $2 \mathrm{keV}$ to $4 \mathrm{keV}$. Above $5 \mathrm{keV}$, the resolution starts to increased. This effect is due to the fact that there is less forward scattering of primary electrons before reaching the QD level. This effect can be seen in material with very short diffusion length. In the case of the sample used in this work, the diffusion can be very important $(\sim 1 \mu \mathrm{m})$. Figure 4 , present a series of absorbed energy images simulated using the previous conditions with at fixed primary energy $(5 \mathrm{keV})$ as a function of the diffusion spheres diameter. This shows that the diffusion of carriers is clearly impacting the observed resolution and by comparing with Monte Carlo simulation could be determined.

\section{References}

D. Drouin and A.R. Couture, Microscopy and Microanalysis, $\underline{8}$, Suppl. 2, 702CD, (2002). 
D. Drouin, A.R. Couture, R. Gauvin, P. Horny, Scanning, Vol. 23, 2, 93-94, (2001).

D. Drouin, P. Hovington et R. Gauvin, Scanning, 19, 20-28, (1997)

D. Joy and S. Luo, Scanning, 11, 176-180, (1989)

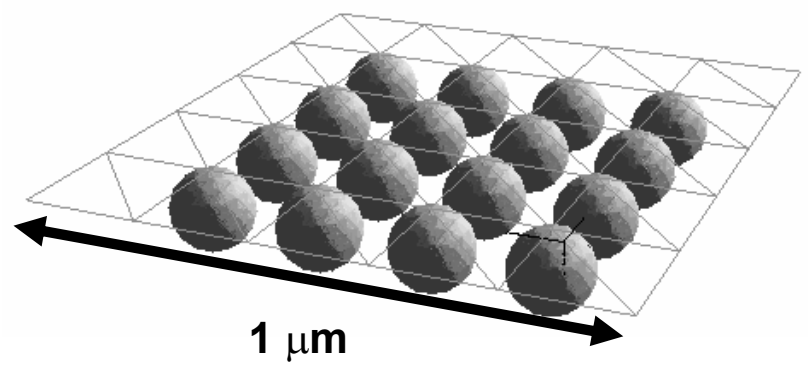

Figure 1 Demonstration sample composed InAs spheres embedded in InP matrix.

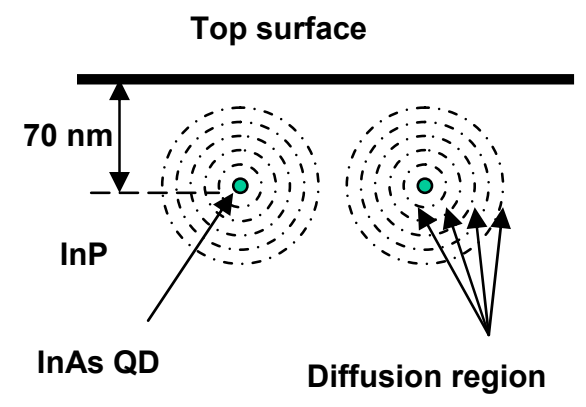

Figure 2 Cross sectional view of 2 QD shown in Figure 1. The chemical composition of all the diffusion regions is the same as the substrate.
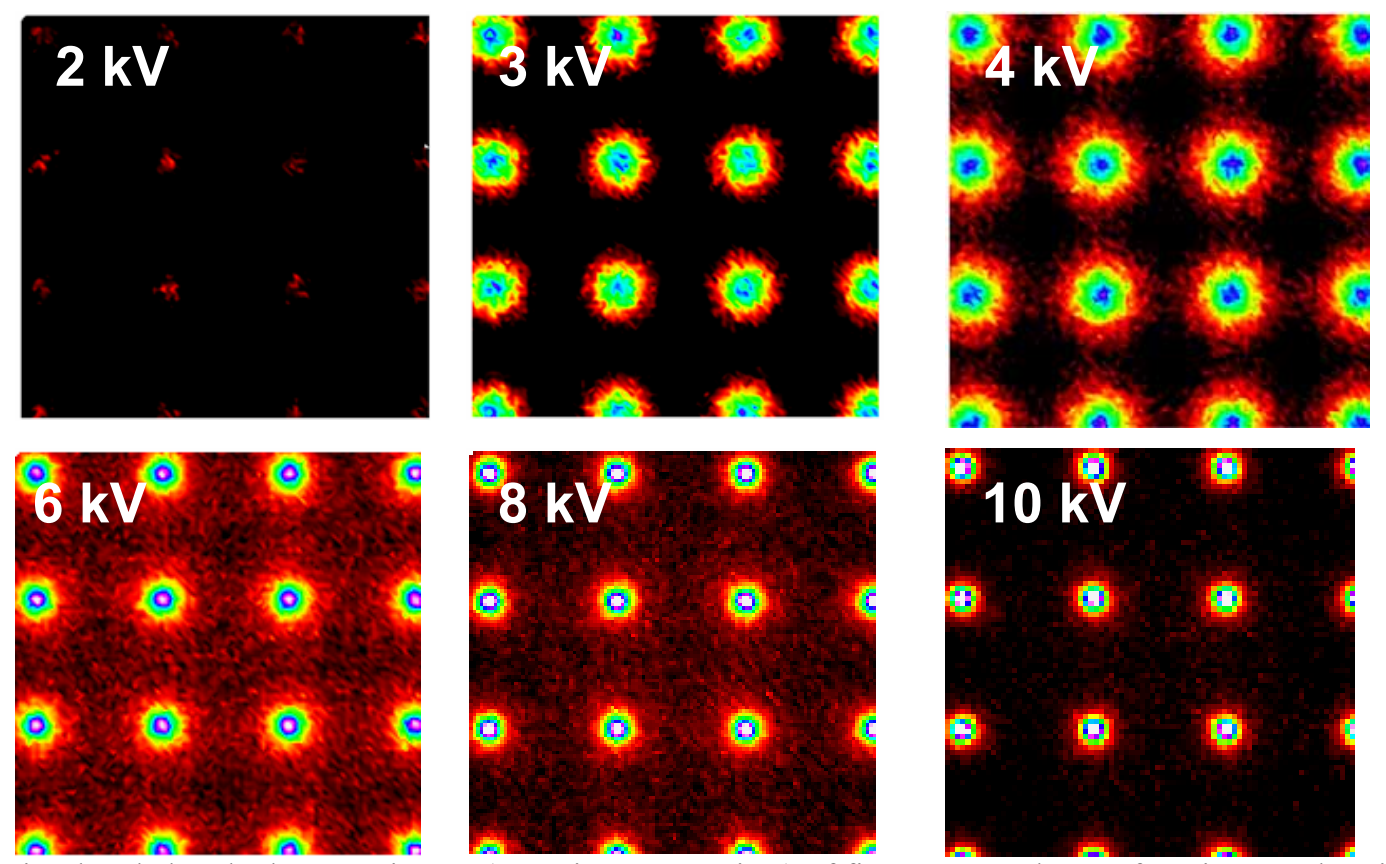

Figure 3 Simulated absorbed energy image (50 points x 50 points) of figure 1 sample as a function accelerating voltage. Number of simulated electrons per points 2000. Total field width $1 \mu \mathrm{m}$.
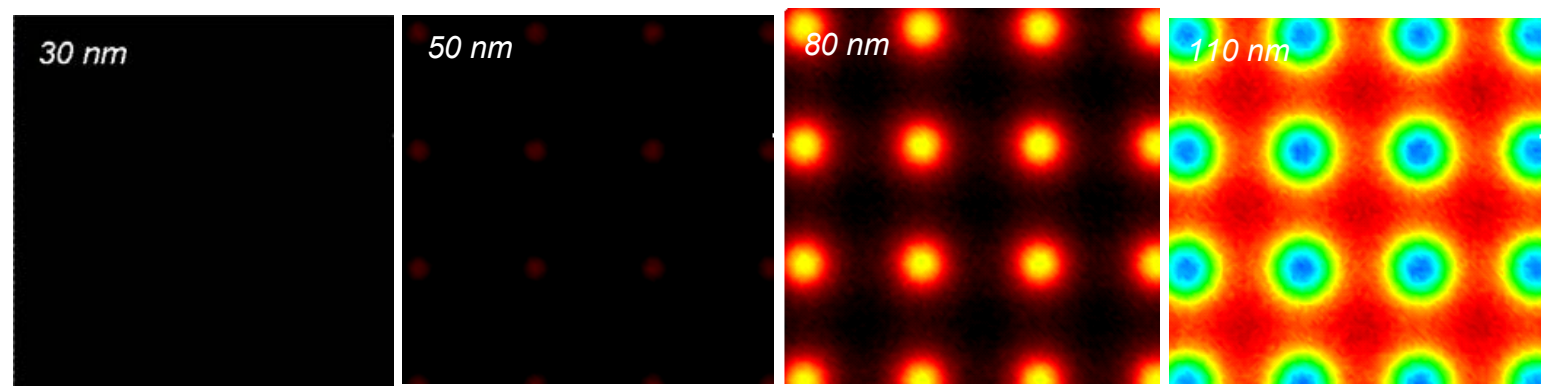

Figure 4 Simulated absorbed energy image (50 points x 50 points) of figure 1 sample as a function of diffusion sphere diameter. Number of simulated electrons per points 2000 , accelerating voltage $5 \mathrm{kV}$. Total field width $1 \mu \mathrm{m}$. 